\title{
Evaluation of targeted antimicrobial prophylaxis for transrectal ultrasound guided prostate biopsy: a prospective cohort trial
}

Teresa R. Zembower ${ }^{1}$, Kelly M. Maxwell2 ${ }^{2}$ Robert B. Nadler², John Cashy², Marc H. Scheetz ${ }^{3,4}$, Chao Qi ${ }^{5}$ and Anthony J. Schaeffer ${ }^{2 *}$

\begin{abstract}
Background: We evaluated the effectiveness of targeted antimicrobial prophylaxis in transrectal ultrasound guided prostate biopsy (TRUSP).

Methods: A prospective, non-randomized cohort study was conducted. Rectal swab cultures plated on non-selective blood agar and on selective MacConkey agar supplemented with ciprofloxacin identified ciprofloxacin-susceptible and -resistant gram-negative bacteria (CS-GNB and CR-GNB). Patients with CS-GNB received ciprofloxacin while those with CR-GNB received directed prophylaxis. Infectious complications were defined clinically and microbiologically within 30 days after TRUSP. Data were derived at 7 and 30 days post procedure by questionnaires and electronic medical records. We hypothesized that there would be no difference in the infectious outcomes among the CS and CR groups.

Results: From November 1, 2012 to March 31, 2015, 510 men completed the study; 430 (84.3\%) had CS-GNB and 80 (15.7\%) had CR-GNB. 484 (94.9\%) completed the study per protocol, while 26 (5.1\%) had an intention-to-treat (ITT) analysis. Of the 484,475 (98.1\%) had no infections, nine (1.9\%) had infections, six of which (1.2\%) were culture-proven (CP). The nine infections were as follows: five (1.0\%) uncomplicated UTIs, one (0.2\%) complicated UTI, and three (0.6\%) urosepsis. One case of uncomplicated UTI and two cases of urosepsis were not CP, but were diagnosed clinically. ITT outcomes were similar. The infection rates were not statistically different between the CS-and CR-GNB patients ( $p$-value $=0.314 ; 95 \% \mathrm{Cl} 0.8-3.3)$. The four patients with complicated UTIs or sepsis were hospitalized for a mean of 2.6 days and discharged without sequelae. Of the nine infections, three were antimicrobial prophylaxis failures (two ciprofloxacin and one amikacin); three were likely due to failure of the collection or processing of the rectal swab or increasing bacterial resistance between the time of swab collection and biopsy, and three developed clinical infections with no isolate recovered.
\end{abstract}

Conclusions: Targeted antimicrobial prophylaxis follows the principles of antimicrobial stewardship and achieved a low rate of infectious complications with limited morbidity and no sequelae. This individualized method of prophylaxis may be widely applied. Further studies are needed to explore reasons for targeted prophylaxis failure and to determine comparative efficacy of non-ciprofloxacin-containing targeted prophylaxis regimens.

Trial registration: ClinicalTrials.gov. NCT01659866. Registered 9 July 2012. First patient enrolled 1 November 2012.

Keywords: Urology, Biopsy, Infection, Antibacterial agents

\footnotetext{
* Correspondence: ajschaeffer@northwestern.edu

${ }^{2}$ Department of Urology, Northwestern University Feinberg School of

Medicine, 303 E. Chicago Ave., Tarry 16-703, Chicago, IL 60611, USA

Full list of author information is available at the end of the article
} 


\section{Background}

More than two million transrectal ultrasound guided prostate biopsies (TRUSP) are performed in the US and Europe annually $[1,2]$. Infectious complications range from uncomplicated urinary tract infections (UTIs) to prostatitis to sepsis and death [3]. Empiric antimicrobial prophylaxis reduced the risk of infectious complications [4] but, with increasing resistance, infections after biopsy have risen [1,5-9] and are most commonly fluoroquinolone (FQ)-resistant (R) gram-negative bacteria (GNB) [10].

Two primary prophylaxis strategies have emerged to prevent post-biopsy infections, a targeted or an empiric approach [7, 11-13]. The targeted approach involves obtaining a pre-biopsy rectal swab culture and choosing an antimicrobial agent based on culture results. The empiric approach is frequently based on local antibiograms [7, 12, 14-16]. Both approaches can be augmented by adding an antimicrobial agent to a fluoroquinolone or combination non-quinolone based regimen. To date, although studies have shown significant reduction in infectious complications, these studies may have been subject to underreporting because they were retrospective $[5,12,14$, 15], did not use phone contact for follow-up [7, 12-16], did not report non-hospitalized infections [7, 11, 12], and followed patients for less than 30 days $[11,16]$. Additionally, the augmented approach introduces the concern for driving further antimicrobial resistance [17].

In our study, we prospectively evaluated patients by phone interview at 7 and 30 days to evaluate the rate of all post-biopsy infections in our patients treated with targeted prophylaxis based on rectal swab culture. Targeted antimicrobial prophylaxis achieved a low rate of infectious complications, limited morbidity and no sequelae in patients with either ciprofloxacin- susceptible or -resistant gram-negative rectal flora. These results suggest that this individualized method of prophylaxis may be widely applied.

\section{Methods}

\section{Study setting and design}

The study was conducted in the Northwestern University (NU) Feinberg School of Medicine Department of Urology at Northwestern Memorial Hospital between November 1, 2012 and March 31, 2015. This prospective, non-randomized cohort trial evaluated the efficacy of targeted pre-procedural antimicrobial prophylaxis for TRUSP and tested the hypothesis that infectious complication rates for targeted prophylaxis in patients with ciprofloxacin-susceptible compared to ciprofloxacin-resistant gram-negative bacteria (CSGNB and CR-GNB, respectively) rectal flora would be equivalent. The Institutional Review Board of NU approved this study.

\section{Recruitment and eligibility criteria}

Eligible patients were men 18 years or older selected to undergo TRUSP to evaluate for prostate cancer. Patients were excluded from the study if (1) they did not complete or withdrew informed consent; (2) their rectal swab cultures were CS-GNB but they did not receive ciprofloxacin as pre-procedure prophylaxis (i.e. ciprofloxacin-allergic patients); (3) their rectal swab cultures showed CR-GNB but they received ciprofloxacin; or (4) they did not complete the pre-biopsy questionnaire or the two post-biopsy phone screening evaluations. Of the 510 study participants, 26, who fulfilled eligibility criteria but received augmented prophylaxis (24 empiric, 2 directed) at the discretion of the treating physician, were evaluated in a separate intention-to-treat (ITT) analysis.

\section{Clinical specimen processing}

After completing a pre-biopsy questionnaire (Additional file 1) to record demographics and to evaluate risk factors for infection, all subjects had rectal swab cultures obtained no more than 30 days prior to the TRUSP (Additional file 2). Swabs were cultured on blood agar and on MacConkey agar supplemented with $1 \mu \mathrm{g} / \mathrm{ml}$ ciprofloxacin (Thermo Scientific ${ }^{\mathrm{Tm}}$ Remel $^{\mathrm{Tm}}$, Waltham, MA, USA). Quality control (QC) was performed on each new lot and shipment of MacConkey agar according to manufacturer's instructions [18]. The following QC strains were utilized: Staphylococcus aureus ATCC ${ }^{\circ}$ 25,923; Pseudomonas aeruginosa ATCC 27,853; Escherichia coli ATCC $^{\circ}$ 25,922; and Escherichia coli strain \#OC110. The plates were placed in a $\mathrm{CO}_{2}$ incubator $\left(35^{\circ}\right.$ to $\left.37^{\circ} \mathrm{C}\right)$ and read at $36-48 \mathrm{~h}$.

\section{Bacterial species identification and antimicrobial susceptibility testing}

Blood agar plates without growth indicated inadequate specimen collections. Specimens with growth on blood agar only were considered to contain CS-GNR. Specimens with growth of gram-negative rods (GNRs) on blood and supplemented MacConkey agar underwent organism identification and antimicrobial susceptibility testing using an automated microbial system (Vitek ${ }^{\circ}$, Biomérieux, Durham, NC, USA) using the AST-GN47 cards. Antimicrobial susceptibilities were reported according to Clinical Laboratory Standards Institute (CLSI) guidelines [19].

\section{Selection of antimicrobial prophylaxis}

Prophylactic antimicrobial agents were selected using a pre-determined protocol (Additional file 3). Patients with CS-GNR received ciprofloxacin $500 \mathrm{mg}$ orally $2 \mathrm{~h}$ before TRUSP and $500 \mathrm{mg}$ orally $12 \mathrm{~h}$ later. Subjects harboring CR-GNB received an antimicrobial agent 
based on the AUA guidelines (Additional file 3). Selection of the narrowest spectrum agent available was encouraged. Twenty-six patients (24 CS, 2 CR) received targeted prophylaxis augmented by another antimicrobial at the discretion of the treating physician based on the patient's clinical status.

\section{Clinical evaluation}

The data were derived from the pre-biopsy questionnaire and electronic medical record review. Charlson score was calculated by ICD-9 codes [20]. Immediately prior to the biopsy, enema and antimicrobial prophylaxis, including drug, dose, and timing, were confirmed. Phone screening at days 7 and 30 following TRUSP determined whether the patients experienced infectious complications (Additional file 4) or adverse drug reactions based on pre-determined criteria.

\section{Outcomes and definitions}

The primary outcome was to compare the rate of infection following TRUSP in subjects with and without CRGNB. Secondary objectives included determination of risk factors for infection and antimicrobial resistance traits of rectal swab isolates. Infectious complications were clinically defined as 1) uncomplicated urinary tract infection (UTI): dysuria, urgency, frequency or hematuria without fever and with or without pyuria ( $>5$ white blood cells per high-powered field or positive leukocyte esterase on urine dipstick) or bacteriuria ( $\geq 10^{5}$ colony-forming units $/ \mathrm{mL}$ ); 2) complicated UTI: fever, flank pain, nausea or vomiting with or without pyuria and bacteriuria; 3) urosepsis: criteria for sepsis, severe sepsis, and septic shock [21] were combined and categorized as urosepsis.

\section{Statistical analysis}

Analyses were performed with SAS version 9.3 (SAS Institute Inc., Cary, NC) and R version 3.3 (R Foundation for Statistical Computing, Vienna, Austria). Categorical variables were described by percentages and compared using Chi-square or Fisher exact tests as appropriate. Continuous variables were described by means and evaluated using Student t-test. A $p$-value $<0.05$ was considered statistically significant. Our initial goal was to enroll 1700 patients, and have enough patients in each group to obtain a $95 \%$ confidence interval for the difference in the complication rates between the two groups with a margin of error of, at most, $2 \%$.

\section{Results}

\section{Clinical characteristics}

Five hundred ten (90.6\%) patients were included in the analysis (Fig. 1). 24 patients underwent more than one

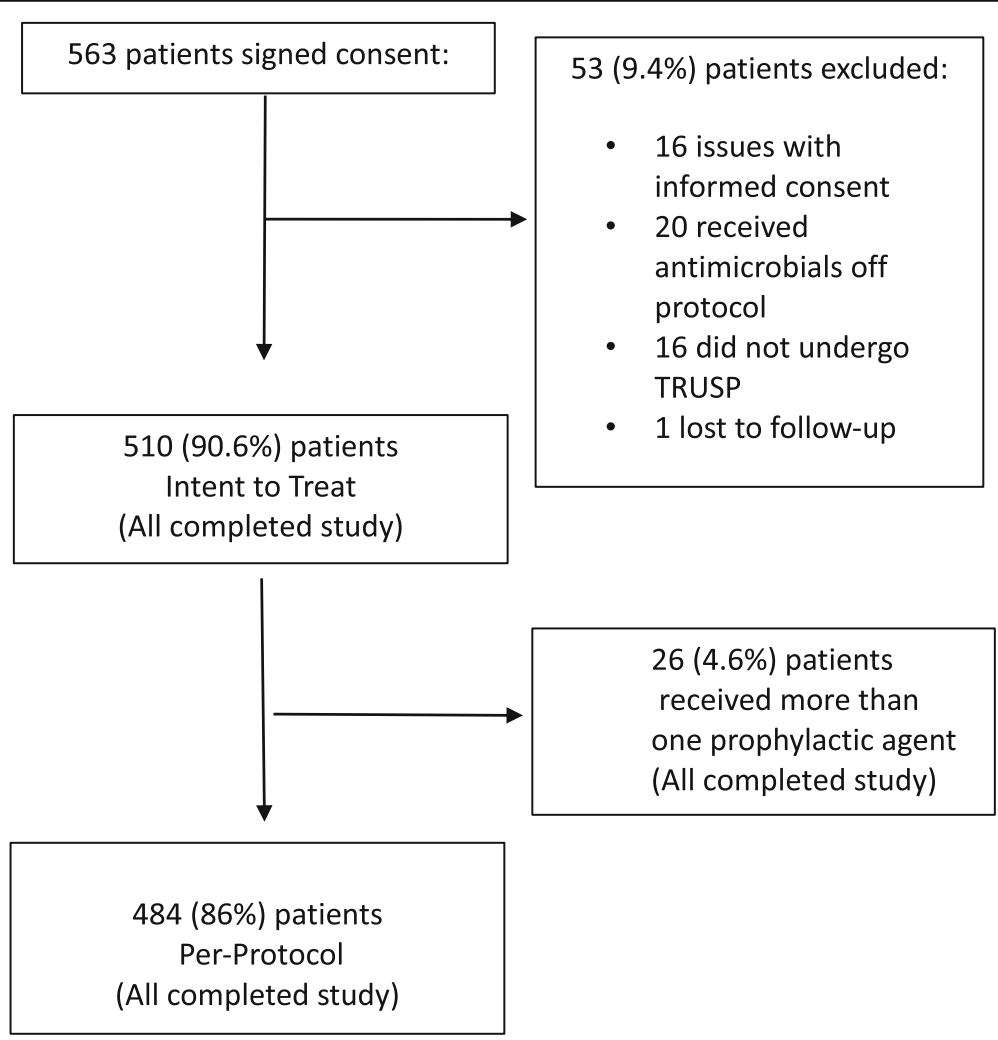

Fig. 1 Study Subjects. TRUSP, Transrectal ultrasound guided prostate biopsy 
Table 1 Demographics and clinical characteristics of the intent to treat study population stratified by ciprofloxacin susceptibility status

\begin{tabular}{|c|c|c|c|c|}
\hline & $\begin{array}{l}\text { Ciprofloxacin-susceptible } \\
N=430(84.3 \%)\end{array}$ & $\begin{array}{l}\text { Ciprofloxacin-resistant } \\
N=80(15.7 \%)\end{array}$ & $\begin{array}{l}\text { All } \\
N=510\end{array}$ & $P$ value \\
\hline \multicolumn{5}{|l|}{ Demographics } \\
\hline Age, mean $+/-S D$, (range), years & $62.7 \pm 9.1(33-88)$ & $61.6 \pm 7.6(42-77)$ & $62.5 \pm 8.9(33-88)$ & 0.323 \\
\hline Race, n (\%) & & & & 0.101 \\
\hline White & $324(75.3)$ & $52(65.0)$ & $376(73.7)$ & \\
\hline Black & $68(15.8)$ & $15(18.8)$ & $83(16.3)$ & \\
\hline Hispanic & $21(4.9)$ & $9(11.2)$ & $30(5.9)$ & \\
\hline Other & $17(4.0)$ & $4(5.0)$ & $21(4.1)$ & \\
\hline \multicolumn{5}{|l|}{ Clinical characteristics } \\
\hline Reason for biopsy, n (\%) & & & & 0.745 \\
\hline Elevated PSA & $357(83.0)$ & $66(82.5)$ & $423(82.9)$ & \\
\hline Abnormal DRE & $29(6.7)$ & $4(5.0)$ & $33(6.5)$ & \\
\hline Both & $27(6.3)$ & $5(6.2)$ & $32(6.3)$ & \\
\hline Other & $17(4.0)$ & $5(6.2)$ & $22(4.3)$ & \\
\hline Biopsy result, n (\%) & & & & 0.848 \\
\hline Negative & $219(50.9)$ & $38(47.5)$ & $257(50.4)$ & \\
\hline Prostate cancer & $167(38.8)$ & $34(42.5)$ & $201(39.4)$ & \\
\hline HGPIN & $44(10.2)$ & $8(10.0)$ & $52(10.2)$ & \\
\hline History of urinary tract infection, $\mathrm{n}(\%)$ & & & & 0.004 \\
\hline Yes & $39(9.1)$ & $16(20.0)$ & $55(10.8)$ & \\
\hline No & $382(88.8)$ & $60(75.0)$ & $442(86.7)$ & \\
\hline Unknown/missing & $9(2.1)$ & $4(5.0)$ & $13(2.5)$ & \\
\hline History of urinary retention; n (\%) & & & & 0.769 \\
\hline Yes & $33(7.7)$ & $6(7.5)$ & $39(7.6)$ & \\
\hline No & $354(82.3)$ & $64(80.0)$ & $418(82.0)$ & \\
\hline Unknown/missing & $43(10.0)$ & $10(12.5)$ & $53(10.4)$ & \\
\hline FQ usage in prior 2 years, n (\%) & & & & 0.921 \\
\hline Yes & $95(22.1)$ & $19(23.8)$ & $114(22.3)$ & \\
\hline No & $225(52.3)$ & $42(52.5)$ & $267(52.4)$ & \\
\hline Unknown/missing & $110(25.6)$ & $19(23.8)$ & $129(25.3)$ & \\
\hline Hospitalized in prior 1 year; n (\%) & & & & 0.653 \\
\hline Yes & $42(9.8)$ & $6(7.5)$ & $48(9.4)$ & \\
\hline No & $374(87.0)$ & $73(91.2)$ & $447(87.7)$ & \\
\hline Unknown/missing & $14(3.3)$ & $1(1.2)$ & $15(2.9)$ & \\
\hline Healthcare worker; n (\%) & & & & 0.728 \\
\hline Yes & $14(3.3)$ & $2(2.5)$ & $16(3.1)$ & \\
\hline No & $357(83.0)$ & $70(87.5)$ & $427(83.7)$ & \\
\hline Unknown/missing & $59(13.7)$ & $8(10.0)$ & $67(13.1)$ & \\
\hline Charlson comorbidity score; n (\%) & & & & 0.693 \\
\hline 0 & $348(80.9)$ & $68(85.0)$ & $416(81.6)$ & \\
\hline 1 & $16(3.7)$ & $2(2.5)$ & $18(3.5)$ & \\
\hline 2 & $32(7.4)$ & $3(3.8)$ & $35(6.9)$ & \\
\hline $3-16$ & $34(7.9)$ & $7(8.8)$ & $41(8.0)$ & \\
\hline
\end{tabular}




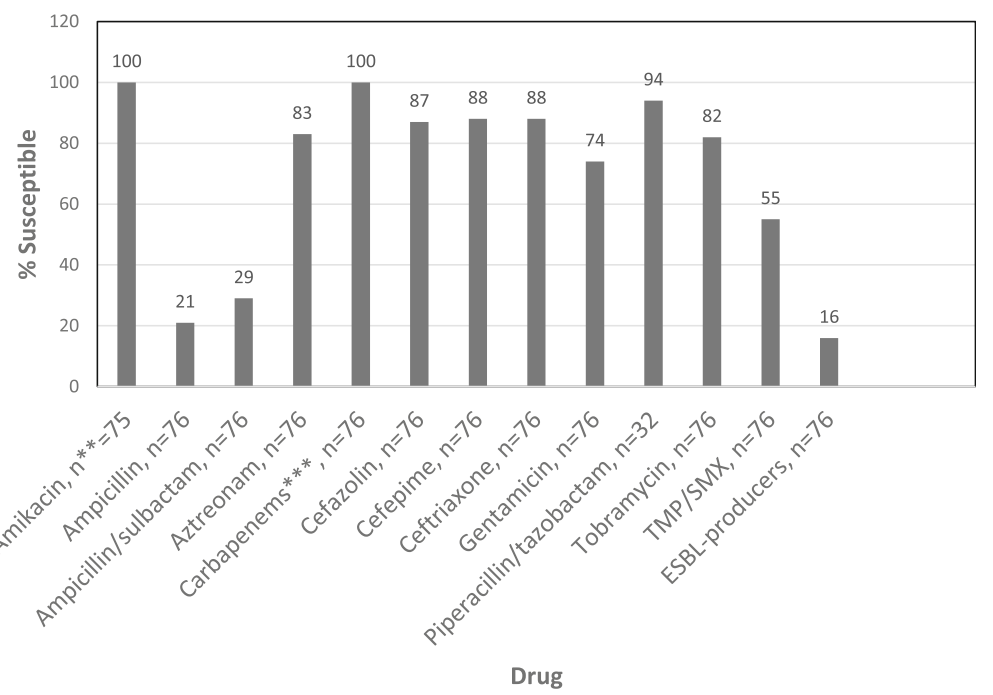

Fig. 2 Antimicrobial Susceptibility Profile of $76^{*}$ Ciprofloxacin-resistant E. coli Isolates. Tmp/smx, Trimethoprim/Sulfamethoxazole. *Not all isolates were tested for all antimicrobial agents, ${ }^{* *}$ Number of isolates tested for this particular antimicrobial agent, ${ }^{* * *}$ Carbapenems tested included imipenem $(n=49)$, meropenem $(n=76)$

biopsy. Outcomes of all were recorded, but only infectious outcomes from the first biopsy were included. The time between rectal swab culture and TRUSP ranged from 7 to 30 days. Of the 510 patients, 26 received a second prophylactic antimicrobial agent at the discretion of the treating physician based on the patient's clinical status; therefore, 510 patients were included in the ITT analysis and 484 in the per-protocol analysis.

Patient demographics and clinical characteristics of the 510 ITT patients stratified by ciprofloxacin susceptibility status are listed in Table 1. In this cohort, 430 (84.3\%) had CS-GNB and 80 (15.7\%) had CR-GNB on rectal swab culture. Univariate analysis demonstrated that patients with $\mathrm{CS}$ and CR rectal flora were similar except that a history of urinary tract infection was more common in $\mathrm{CR}$ patients $(p=0.004)$. Similar results were observed in the per-protocol population. (Data not shown).

\section{Microbiological characteristics}

Of the 80 patients with CR-GNB, 76 (95\%) harbored Escherichia coli, 2 (2.6\%) Pseudomonas aeruginosa, 1 (1.2\%) Citrobacter freundii and 1 (1.2\%) Klebsiella pneumoniae. The antimicrobial susceptibility profiles of the CR-E. coli isolates are shown in Fig. 2. Using the Centers for Disease Control and Prevention (CDC) definitions [22] to classify the bacteria, $62(79.5 \%)$ were multidrugresistant (MDR), 17 (21.5\%) were not MDR, and only 12 (15.2\%) were resistant to ciprofloxacin alone. Of the MDR E. coli, 12 (15.2\%) were extended-spectrum betalactamase producers (ESBLs). Interestingly, of the 24 patients who underwent two biopsies during the study period, the ciprofloxacin susceptibility status of 23 patients remained the same, while one patient who originally had CR-GNB had CS-GNB on subsequent culture. The antimicrobial prophylaxis regimens received are listed in Table 2. Oral regimens were used for 61 (76.3\%) of the 80 patients harboring CR-GNB. Twentyfour patients with CS-GNB received prophylaxis with ciprofloxacin plus another empiric antimicrobial agent; likewise, 2 with CR-GNB received prophylaxis with two non-FQ agents directed by rectal flora susceptibility.

\section{Infectious complications}

The clinical characteristics stratified by infectious outcomes for the ITT and the per-protocol patients are

Table 2 Antimicrobial prophylaxis received versus ciprofloxacin resistance status

\begin{tabular}{lll}
\hline $\begin{array}{l}\text { Drug received } \\
N=510\end{array}$ & $\begin{array}{l}\text { Ciprofloxacin-susceptible } \\
430,(84.3 \%)\end{array}$ & $\begin{array}{l}\text { Ciprofloxacin-resistant } \\
80,(16.3 \%)\end{array}$ \\
\hline Amikacin & 0 & $2(2.5)$ \\
Aztreonam & 0 & $1(1.2)$ \\
Bactrim & 0 & $23(28.8)$ \\
Bactrim + Gentamicin & 0 & $1(1.2)$ \\
Bactrim + Meropenem & 0 & $1(1.2)$ \\
Ceftriaxone & 0 & $3(3.8)$ \\
Cefuroxime & 0 & $38(47.5)$ \\
Ciprofloxacin & $406(94.4)$ & 0 \\
Ciprofloxacin + & $3(0.7)$ & 0 \\
Amoxicillin & & 0 \\
Ciprofloxacin + & $21(4.9)$ & $4(5.0)$ \\
Gentamicin & & $7(8.8)$ \\
Ertapenem & 0 & \\
Gentamicin & 0 &
\end{tabular}


shown in Tables 3 and 4, respectively. In the ITT group, 501 patients $(98.2 \%)$ did not develop infections, while 9 (1.8\%) had clinical infections and $6(1.2 \%)$ of these were culture-proven (CP). In the per-protocol group, 475 patients (98.1\%) did not develop infections, while 9 (1.9\%) had clinical infections and again, 6 (1.2\%) of these were CP. No statistically significant differences were observed in the demographics, clinical characteristics, or infection rate of the ITT versus the perprotocol analysis.

The infection outcomes stratified by CS or CR status for the ITT and per-protocol groups are shown in Table 5. All

Table 3 Demographic and clinical characteristics stratified by infection outcomes in the intent to treat study population

\begin{tabular}{|c|c|c|c|c|}
\hline & $\begin{array}{l}\text { No infection } \\
N=501(98.2 \%)\end{array}$ & $\begin{array}{l}\text { Infection } \\
N=9(1.8 \%)\end{array}$ & $\begin{array}{l}\text { All } \\
N=510\end{array}$ & $P$ value \\
\hline \multicolumn{5}{|l|}{ Demographics } \\
\hline Age, mean $+/-S D$, (range), years & $62.5 \pm 8.9(33-88)$ & $63.4 \pm 6.5(48-71)$ & $62.5 \pm 8.9(33-88)$ & 0.75 \\
\hline Race, n (\%) & & & & 1.0 \\
\hline White & $368(73.5)$ & $8(88.9)$ & $376(73.7)$ & \\
\hline Black & $82(16.4)$ & $1(11.1)$ & $83(16.3)$ & \\
\hline Hispanic & $30(6.0)$ & $0(0.0)$ & $30(5.9)$ & \\
\hline Other & $21(4.2)$ & $0(0.0)$ & $21(4.1)$ & \\
\hline \multicolumn{5}{|l|}{ Clinical characteristics } \\
\hline Biopsy result, n (\%) & & & & 0.611 \\
\hline Negative & $253(50.5)$ & $4(44.4)$ & $257(50.4)$ & \\
\hline Prostate cancer & $196(39.1)$ & $5(55.6)$ & $201(39.4)$ & \\
\hline HGPIN & $52(10.4)$ & $0(0.0)$ & $52(10.2)$ & \\
\hline History of urinary tract infection, $n(\%)$ & & & & 0.029 \\
\hline Yes & $52(10.4)$ & $3(33.3)$ & $55(10.8)$ & \\
\hline No & $437(87.2)$ & $5(55.6)$ & $442(86.7)$ & \\
\hline Unknown/missing & $12(2.4)$ & $1(11.1)$ & $13(2.5)$ & \\
\hline History of urinary retention; n (\%) & & & & 0.499 \\
\hline Yes & $38(7.6)$ & $1(11.1)$ & $39(7.6)$ & \\
\hline No & $410(81.8)$ & $8(88.9)$ & $418(82.0)$ & \\
\hline Unknown/missing & $53(10.6)$ & $0(0.0)$ & $53(10.4)$ & \\
\hline FQ usage in prior 2 years, $\mathrm{n}(\%)$ & & & & 0.678 \\
\hline Yes & $111(22.2)$ & $3(33.3)$ & $114(22.3)$ & \\
\hline No & $263(52.5)$ & $4(44.4)$ & $267(52.4)$ & \\
\hline Unknown/missing & $127(25.3)$ & $2(22.2)$ & $129(25.3)$ & \\
\hline Hospitalized in prior 1 year; $n$ (\%) & & & & 0.400 \\
\hline Yes & $46(9.2)$ & $2(22.2)$ & $48(9.4)$ & \\
\hline No & $440(87.8)$ & $7(77.8)$ & $447(87.7)$ & \\
\hline Unknown/missing & $15(3.0)$ & $0(0.0)$ & $15(2.9)$ & \\
\hline Healthcare worker; n (\%) & & & & 0.713 \\
\hline Yes & $16(3.2)$ & $0(0.0)$ & $16(3.1)$ & \\
\hline No & $418(83.4)$ & $9(100)$ & $427(83.7)$ & \\
\hline Unknown/missing & $67(13.4)$ & $0(0.0)$ & $67(13.1)$ & \\
\hline Charlson comorbidity score; n (\%) & & & & 0.139 \\
\hline 0 & $410(81.8)$ & $6(66.7)$ & 416 (81.6) & \\
\hline 1 & $17(3.4)$ & $1(11.1)$ & $18(3.5)$ & \\
\hline 2 & $35(7.0)$ & $0(0.0)$ & $35(6.9)$ & \\
\hline $3-16$ & $39(7.8)$ & $2(22.2)$ & $41(8.0)$ & \\
\hline
\end{tabular}


Table 4 Demographics and clinical characteristics stratified by infection outcomes in the per-protocol study population

\begin{tabular}{|c|c|c|c|c|}
\hline & $\begin{array}{l}\text { No infection } \\
N=475 \text { (98.1\%) }\end{array}$ & $\begin{array}{l}\text { Infection } \\
N=9(1.9 \%)\end{array}$ & $\begin{array}{l}\text { All } \\
N=484\end{array}$ & $P$ value \\
\hline \multicolumn{5}{|l|}{ Demographics } \\
\hline Age, mean $\pm S D$, (range), years & $62.5 \pm 9.0(33-88)$ & $63.4 \pm 6.5(48-71)$ & $62.5 \pm 9.0(48-71)$ & 0.75 \\
\hline Race, n (\%) & & & & 1.0 \\
\hline White & $351(73.9)$ & $8(88.9)$ & $359(74.2)$ & \\
\hline Black & $77(16.2)$ & $1(11.1)$ & $78(16.1)$ & \\
\hline Hispanic & $27(5.7)$ & $0(0.0)$ & $27(5.6)$ & \\
\hline Other & $20(4.2)$ & $0(0.0)$ & $20(4.1)$ & \\
\hline \multicolumn{5}{|l|}{ Clinical characteristics } \\
\hline Biopsy result, n (\%) & & & & 0.609 \\
\hline Negative & $241(50.7)$ & $4(44.4)$ & $245(50.6)$ & \\
\hline Prostate cancer & $185(38.9)$ & $5(55.6)$ & $190(39.3)$ & \\
\hline HGPIN & $49(10.3)$ & $0(0.0)$ & $49(10.1)$ & \\
\hline History of urinary tract infection, $\mathrm{n}(\%)$ & & & & 0.029 \\
\hline Yes & $50(10.5)$ & $3(33.3)$ & $53(10.9)$ & \\
\hline No & $414(87.2)$ & $5(55.6)$ & $419(86.6)$ & \\
\hline Unknown/missing & $11(2.3)$ & $1(11.1)$ & $12(2.5)$ & \\
\hline History of urinary retention; n (\%) & & & & 0.511 \\
\hline Yes & $38(8.0)$ & $1(11.1)$ & $39(8.1)$ & \\
\hline No & $387(81.5)$ & $8(88.9)$ & $395(81.6)$ & \\
\hline Unknown/missing & $50(10.5)$ & $0(0.0)$ & $50(10.3)$ & \\
\hline FQ usage in prior 2 years, $\mathrm{n}(\%)$ & & & & 0.681 \\
\hline Yes & $109(22.9)$ & $3(33.3)$ & $112(23.1)$ & \\
\hline No & $254(53.5)$ & $4(44.4)$ & $258(53.3)$ & \\
\hline Unknown/Missing & $112(23.6)$ & $2(22.2)$ & $114(23.6)$ & \\
\hline Hospitalized in prior 1 year; $n$ (\%) & & & & 0.388 \\
\hline Yes & $44(9.3)$ & $2(22.2)$ & $46(9.5)$ & \\
\hline No & $418(88.0)$ & $7(77.8)$ & $425(87.8)$ & \\
\hline Unknown/missing & $13(2.7)$ & $0(0.0)$ & $13(2.7)$ & \\
\hline Healthcare worker; n (\%) & & & & 0.710 \\
\hline Yes & $16(3.4)$ & $0(0.0)$ & $16(3.3)$ & \\
\hline No & $406(85.5)$ & $9(100.0)$ & $415(85.7)$ & \\
\hline Unknown/missing & $53(11.2)$ & $0(0.0)$ & $53(11.0)$ & \\
\hline Charlson comorbidity score; n (\%) & & & & 0.144 \\
\hline 0 & $387(81.5)$ & $6(66.7)$ & $393(81.2)$ & \\
\hline 1 & $16(3.4)$ & $1(11.1)$ & $17(3.5)$ & \\
\hline 2 & $35(7.4)$ & $0(0.0)$ & $35(7.2)$ & \\
\hline $3-16$ & $37(7.8)$ & $2(22.2)$ & $39(8.1)$ & \\
\hline
\end{tabular}

Abbreviations: HGPIN high-grade prostatic intraepithelial neoplasia, FQ fluoroquinolone

infections occurred in the per-protocol patients. There was no statistically significant difference in the rate of infections ( $\mathrm{CS}=1.5 \%$; $\mathrm{CR}=3.9 \%$; $p=0.337$ ) between the two groups. Additionally, there were no statistically significant differences in the efficacy of the prophylactic regimens used. (Data not shown).
The characteristics of the 9 patients who developed infectious complications are shown in Table 6. All infections occurred in patients who received single drug targeted prophylaxis. Infectious complications occurred in $6(66.7 \%)$ patients within 7 days and in $3(33.3 \%)$ patients at 8,11 , and 13 days, respectively. 
Table 5 Infectious outcomes of the intent to treat and per-protocol study populations stratified by ciprofloxacin susceptibility status

\begin{tabular}{|c|c|c|c|c|}
\hline & Ciprofloxacin-susceptible & Ciprofloxacin-resistant & All & $P$ value \\
\hline Intent to Treat & $N(\%)=430(84.3)$ & $N(\%)=80(15.7)$ & $N(\%)=510$ & \\
\hline Any infection $\mathrm{n},(\%), 95 \% \mathrm{Cl}$ & $6,(1.4), 0.5-3.8$ & $3,(3.8), 0.8-10.6$ & 9 (1.8) 0.8-3.3 & 0.314 \\
\hline No/Yes (n/n; \%/\%) & $424 / 6(98.6 / 1.4)$ & 77/3 (96.2/3.8) & $501 / 9(98.2 / 1.8)$ & \\
\hline Type of infection; n (\%) & & & & 0.277 \\
\hline None & $424(98.6)$ & $77(96.2)$ & $501(98.2)$ & \\
\hline Uncomplicated UTI & $3(0.7)$ & $2(2.5)$ & $5(1.0)$ & \\
\hline Complicated UTI & $1(0.2)$ & $0(0.0)$ & $1(0.2)$ & \\
\hline Urosepsis & $2(0.5)$ & $1(1.3)$ & $3(0.6)$ & \\
\hline Per Protocol & $N=406(83.9 \%)$ & $N=78(16.1 \%)$ & $N=484$ & \\
\hline Any infection $\mathrm{n},(\%), 95 \% \mathrm{Cl}$ & $6,(1.5), 0.5-3.0$ & $3,(3.9), 0.8-10.8)$ & $9,(1.9), 0.9-3.5$ & 0.337 \\
\hline No/Yes (n/n; \%/\%) & $400 / 6(98.5 / 1.5)$ & 75/3 (96.1/3.9) & 475/9 (98.1/1.9) & \\
\hline Type of infection; n (\%) & & & & 0.288 \\
\hline None & $400(98.5)$ & $75(96.1)$ & $475(98.1)$ & \\
\hline Uncomplicated UTI & $3(0.7)$ & $2(2.6)$ & $5(1.0)$ & \\
\hline Complicated UTI & $1(0.2)$ & $0(0.0)$ & $1(0.2)$ & \\
\hline Urosepsis & $2(0.5)$ & $1(1.3)$ & $3(0.6)$ & \\
\hline
\end{tabular}

Abbreviations: UTI urinary tract infection

The 5 patients with uncomplicated UTIs were managed as outpatients, 4 with and 1 without antimicrobial therapy; those with complicated UTIs or sepsis were managed with antimicrobial therapy as inpatients for 15 (mean 2.6) days.

All patients recovered without sequelae. There were no drug-related adverse events. None of the 26 patients in the ITT group who received augmented prophylaxis developed infectious complications.

Three (33.3\%) of the 9 patients with infections were culture negative. Of the 6 patients with positive cultures, 3 (2 ciprofloxacin and 1 amikacin) were prophylaxis failures, i.e. the infecting bacteria were susceptible to the prophylactic drug they received. One patient who received ciprofloxacin was infected with bacteria with a MIC that was intermediate to ciprofloxacin and 2 patients, one who received trimethoprim/sulfamethoxazole and one who received ciprofloxacin, broke through with organisms fully resistant to these drugs.

\section{Discussion}

With empiric single drug antimicrobial prophylaxis for TRUSP the hospitalization rate for infections is 0 to $6.3 \%{ }^{2}$ Two approaches to address this have been developed. Empiric augmented prophylaxis has shown initially promising results. However, it is often influenced by local hospital antibiograms, fails to assess the rectal source of the post-biopsy infections, and its use will likely be directly related to increasing antimicrobial resistance. Alternatively, rectal swab cultures can 1) determine the population of fluoroquinolone-resistant (FQR)-GNB in the rectal flora, 2) identify specific patients with FQ -GNB and 3) guide targeted single drug and augmented prophylaxis $[7,14,16,19,23,24]$. The presence of FQ-R bacteria in the rectal flora constitutes a five-fold increase in the rate and potential severity of post-biopsy infections in patients receiving empiric fluoroquinolone prophylaxis [25]. This finding supports the utilization of pre-biopsy rectal cultures to identify patients at increased risk and to select targeted prophylaxis that is most likely to be effective. These principles were supported by this prospective cohort study, which showed a very low, i.e. $0.6 \%$, sepsis rate and equivalent infectious complication rates among patients with CRGNB or CS-GNB rectal flora who received targeted prophylaxis per our protocol.

In our study, the FQ resistance rate was $15.7 \%$. We agree with Van Besien et al. [25] who stated that the benefit of targeted prophylaxis depends on local FQ-R prevalence rates. A randomized, blinded trial would subject the approximately $20 \%$ of patients who harbor FQ-R flora and receive FQ prophylaxis to the known 5-fold higher risk of infectious complications [25]. Similarly, empiric augmented prophylaxis could also subject patients to ineffective antimicrobial prophylaxis. For example, gentamicin is frequently used for augmented prophylaxis, but gentamicin resistance was present in $20 \%$ of the bacteria isolated from our patients with CRGNB rectal flora (Fig. 2). 


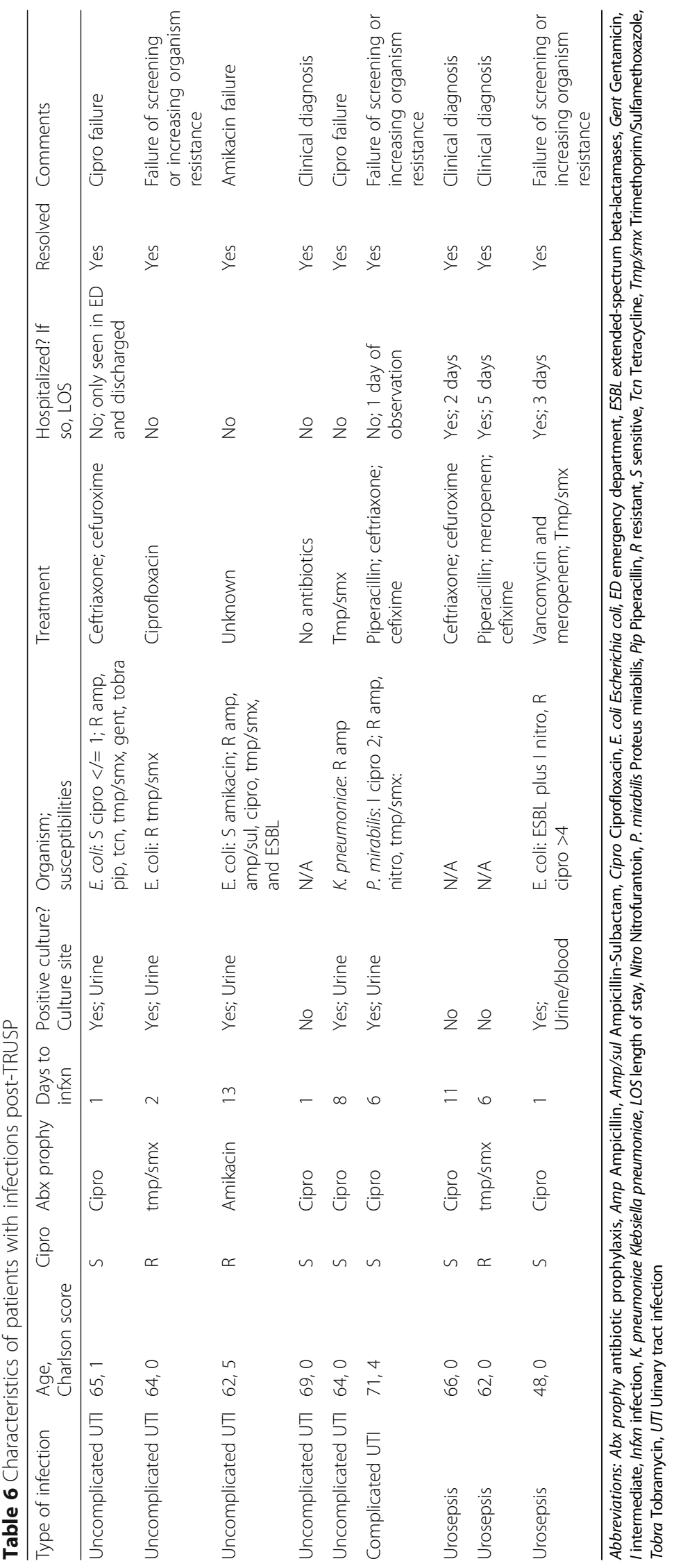


Our infectious complication rate in the per-protocol patients of $1.9 \%$ improved upon the $2.6 \%$ infection rate prior to the introduction of this protocol [14]. We used very stringent criteria: inclusion of all patients with symptoms of urinary tract infection irrespective of urine or blood cultures and phone screening at 7-and 30-days. Indeed, of the 9 patients identified, 3 had negative cultures before antimicrobial therapy was initiated and 3 were negative at 7 days but positive within 30 days. More importantly, only 4 ( $0.8 \%)$ had significant clinical infections and only 2 were culture-proven, febrile UTI $(0.2 \%)$ and sepsis $(0.2 \%)$.

Of the 9 patients with infections, only 3 had prior exposure to fluoroquinolones, a rate similar to those who did not become infected. Of the 6 patients with positive cultures, 3 were prophylaxis failures, i.e. the infecting bacteria was susceptible to the prophylactic drug given, (ciprofloxacin 2, and amikacin 1). Since all of these patients received their prophylaxis per protocol, we cannot implicate noncompliance of the patient or medical error. Breakthrough infections were probably due to excessive inoculum at the time of biopsy and/or an unrecognized host risk factor(s). Three patients were infected with bacteria with antimicrobial susceptibility breakpoints that showed resistance whereas the pre-biopsy rectal bacteria were susceptible. This could be due to sampling error of the rectal flora, lack of detection on the selective media or increasing antimicrobial resistance between the time of the culture and the biopsy. Although incorporating a lower concentration of ciprofloxacin into the screening media may have rendered the two rectal cultures with intermediate susceptibility ciprofloxacinresistant, this extremely low incidence in our opinion would not support lowering the ciprofloxacin concentration in the screening media in our population.

Of the 6 culture-proven infections, the bacteria were multidrug resistant in 5, and of these, 2 were ESBLs. Williamson et al. noted similar results [26]. We identified trends in risk factors, e.g. history of UTI, however the incidence of infections was too low to achieve statistical significance [12, 24, 27-29].

This study is limited in that it was a single institution study and, for ethical reasons, was not blinded or controlled. Erectile dysfunction, a potential complication of TRUSP, was not evaluated, but it may occur as a result of inflammation induced by infection [30]. Thus, targeted prophylaxis could have other indirect benefits. Larger, multicenter studies are necessary to study this approach and its generalizability.

Additionally, this study does not claim superiority to empiric augmented prophylaxis in terms of infection reduction. However, it is likely that antimicrobial prophylaxis based on real time sensitivity data will be more durable and will likely outperform empiric prophylaxis as bacterial resistance inevitably increases. It is possible that more extended use of augmented prophylaxis or use of multiday therapy $[16,29,31,32]$ would have reduced our infectious complications further, but adherence to the guidelines of antimicrobial stewardship favors limited, single drug targeted prophylaxis for most patients.

\section{Conclusions}

Targeted antimicrobial prophylaxis achieved a low rate of infectious complications in patients with CSor CR- GNB rectal flora, limited morbidity and no sequelae. These results suggest that this individualized method of prophylaxis may be widely applied. Further studies are needed to explore reasons for targeted prophylaxis failure and to determine comparative efficacy of non-ciprofloxacin-containing targeted prophylaxis regimens.

\section{Additional files}

Additional file 1: Pre-biopsy Questionnaire. This supplementary document details the data obtained from participants prior to biopsy to collect demographic information and to evaluate risk factors for infection. (DOCX $13 \mathrm{~kb}$ )

Additional file 2: Surveillance for Ciprofloxacin Resistant Enterobacteriaceae using MacConkey Agar with 1 mg/ml Ciprofloxacin. This supplementary document describes the protocol used for rectal swab culture in this study. (DOCX 16 kb)

Additional file 3: Antimicrobial Recommendations for TRUSP Prophylaxis. This supplementary document describes the protocol for selection of prophylactic antimicrobial agents. (DOCX $16 \mathrm{~kb}$ )

Additional file 4: Post-biopsy Phone Questionnaire. This supplementary document lists the questions asked of participants via phone following biopsy to assess for infectious complications and adverse drug reactions. (DOCX $71 \mathrm{~kb})$

\section{Abbreviations}

Abx prophy: Antibiotic prophylaxis; Amp: Ampicillin; Amp/sul: AmpicillinSulbactam; AUA: American Urological Association; Cipro: Ciprofloxacin; CLSI: Clinical Laboratory Standards Institute; CP: Culture proven; CRGNB: Ciprofloxacin-resistant gram-negative bacteria; CS-GNB: Ciprofloxacinsensitive gram-negative bacteria; DRE: Digital rectal exam; E. coli: Escherichia coli; ED: Emergency department; ESBL: Extended-spectrum beta-lactamases; FQ: Fluoroquinolone; FQ-R: Fluoroquinolone-resistant; Gent: Gentamicin; GNR: Gram-negative rods; HGPIN: High-grade prostatic intraepithelial neoplasia; I: Intermediate; Infxn: Infection; ITT: Intent to treat; K. pneumonia: Klebsiella pneumoniae; LOS: Length of stay; MDR: Multi-drug resistant; Nitro: Nitrofurantoin; NU: Northwestern University; P. mirabilis: Proteus mirabilis; Pip: Piperacillin; PSA: Prostate-specific antigen; R: Resistant; S: Sensitive; Tcn: Tetracycline; Tmp/ smx: Trimethoprim/Sulfamethoxazole; Tobra: Tobramycin; TRUSP: Transrectal ultrasound guided prostate biopsy; UTI: Urinary tract infection

\section{Acknowledgements}

The authors would like to thank the research study coordinators and members of the Department of Urology who contributed to this study: Mary Kate Keeter MPH, Annette Kinsella RN, Darlene Marko RN, William Catalona MD, Daniel Dalton MD, John Garnett MD, Stephanie Kielb MD, James Kozlowski MD, Shilajit Kundu MD, William Lin MD, and Joshua Meeks MD/PhD. 


\section{Funding}

The State of Illinois Excellence in Academic Medicine (EAM) grant funded this project: STU00059558 EAM-237. Support was provided for data collection, study design and interpretation. The funding body had no influence on these activities.

\section{Availability of data and materials}

The datasets generated and analyzed during the current study are available from the corresponding author. Potential collaborators or request for data can be submitted to ajschaeffer@northwestern.edu.

\section{Authors' contributions}

The study was conceived by AJS and TZ. The study was designed by AJS, TZ, JC, MS and CQ. Patient recruitment and study coordination was conducted by KM, RN, TZ, and AJS. Data analysis and interpretation was performed by TZ, JC, AJS, MS and CQ. AJS, TZ, KM and JC drafted the manuscript. All authors read and approved the final manuscript

\section{Competing interests}

The authors declare that they have no competing interests.

\section{Consent for publication}

Not applicable.

\section{Ethics approval and consent to participate}

The Northwestern University Institutional Review Board approved this study (STU00059558). The clinical trial was registered on Clinicaltrials.gov (NCT01659866) prior to the start of the study (Registered 9 July 2012. First patient enrolled 1 November 2012). Written informed consent to participate in the study was obtained from all study participants.

\section{Publisher's Note}

Springer Nature remains neutral with regard to jurisdictional claims in published maps and institutional affiliations.

\section{Author details}

'Department of Medicine, Division of Infectious Diseases, Northwestern University Feinberg School of Medicine, 645 N. Michigan Ave., Suite 900, Chicago, IL 60611, USA. ²Department of Urology, Northwestern University Feinberg School of Medicine, 303 E. Chicago Ave., Tarry 16-703, Chicago, IL 60611, USA. ${ }^{3}$ Department of Pharmacy Practice, Midwestern University Chicago College of Pharmacy, 531 31st St., Alumni Hall 381, Downers Grove, IL 60515, USA. ${ }^{4}$ Department of Pharmacy, Northwestern Memorial Hospital, 251 E. Huron St., Feinberg Pavilion LC 700, Chicago, IL 60611, USA.

${ }^{5}$ Department of Pathology, Clinical Microbiology Division, Northwestern Memorial Hospital, 303 E. Chicago Ave., Ward 2-140, Chicago, IL 60611, USA.

Received: 2 March 2017 Accepted: 16 May 2017

Published online: 07 June 2017

\section{References}

1. Loeb S, Carter HB, Berndt SI, et al. Complications after prostate biopsy: data from SEER-Medicare. J Urol. 2011;186:1830-4.

2. Loeb S, Vellekoop A, Ahmed HU, et al. Systematic review of complications of prostate biopsy. Eur Urol. 2013;64:876-92.

3. Carlson WH, Bell DG, Lawen JG, et al. Multi-drug resistant E.Coli urosepsis in physicians following transrectal ultrasound guided prostate biopsies-three cases including one death. Can J Urol. 2010:17:5135-7.

4. Zani EL, Clark OA, Rodrigues Netto N Jr. Antibiotic prophylaxis for transrectal prostate biopsy. Cochrane Database Syst Rev. 2011; doi:10.1002/14651858.

5. Taylor A, Murphy A, Cashy J, et al. Targeted antimicrobial prophylaxis using rectal swab (RS) cultures in men undergoing transrectal ultrasound guided prostate biopsy (TRUSP) significantly reduces the incidence of post procedure infectious complications and cost of care. Presented at the 2011 American Urological Association Annual Meeting; May 12, 2011; Washington, DC.

6. Nam RK, Saskin R, Lee Y, et al. Increasing hospital admission rates for urological complications after transrectal ultrasound guided prostate biopsy. J Urol. 2010;183:963-8.

7. Womble PR, Linsell SM, Gao Y, et al. A statewide intervention to reduce hospitalizations after prostate biopsy. J Urol. 2015;194:403-9.
8. Carignan A, Roussy JF, Lapointe V, et al. Increasing risk of infectious complications after transrectal ultrasound-guided prostate biopsies: time to reassess antimicrobial prophylaxis? Eur Urol. 2012;62:453-9.

9. Mosharafa AA, Torky MH, El Said WM, et al. Rising incidence of acute prostatitis following prostate biopsy: fluoroquinolone resistance and exposure is a significant risk factor. Urology. 2011;78:511-4.

10. Dalhoff A. Global fluoroquinolone resistance epidemiology and implications for clinical use. Interdiscip Perspect Infect Dis. 2012; doi:10.1155/2012/97627.

11. Adibi $M$, Hornberger $B, B$ hat $D$, et al. Reduction in hospital admission rates due to post-prostate biopsy infections after augmenting standard antibiotic prophylaxis. J Urol. 2013;189:535-40.

12. Liss MA, Kim W, Moskowitz D, et al. Comparative effectiveness of targeted vs empirical antibiotic prophylaxis to prevent sepsis from transrectal prostate biopsy: a retrospective analysis. J Urol. 2015;194:397-402.

13. Gil-Vernet Sedo JM, Alvarez-Vijande GR. Effect of intrarectal povidone-iodine in the incidence of infectious complications after transrectal prostatic biopsy. Arch Esp Urol. 2012;65:463-6.

14. Taylor AK, Zembower TR, Nadler RB, et al. Targeted antimicrobial prophylaxis using rectal swab cultures in men undergoing transrectal ultrasound guided prostate biopsy is associated with reduced incidence of postoperative infectious complications and cost of care. J Urol. 2012;187:1275-9.

15. Dai J, Leone A, Mermel L, et al. Rectal swab culture-directed antimicrobial prophylaxis for prostate biopsy and risk of postprocedure infection: a cohort study. Urology. 2015;85:8-14

16. Duplessis CA, Bavaro M, Simons MP, et al. Rectal cultures before transrectal ultrasound-guided prostate biopsy reduce post-prostatic biopsy infection rates. Urology. 2012;79:556-61.

17. Walker JT, Singla N, Roehrborn CG. Reducing infectious complications following transrectal ultrasound-guided prostate biopsy: a systematic review. Rev Urol. 2016:18:73-89.

18. Remel Technical Manual of Microbiological Media. https://tools.thermofisher com/content/sfs/manuals/IFU1545.pdf. Accessed April 17, 2017.

19. Clinical and Laboratory Standards Institute (CLSI). Performance standards for antimicrobial susceptibility testing; approved standard; 25th informational supplement. CLSI document M100-S25. Clinical and Laboratory Standards Institute, Wayne, PA, 2015

20. Charlson ME, Pompei P, Ales KL, et al. A new method of classifying prognostic comorbidity in longitudinal studies: development and validation. J Chronic Dis. 1987:40:373-83.

21. Levy MM, Fink MP, Marshall JC, et al. 2001 SCCM/ESICM/ACCP/ATS/SIS international sepsis definitions conference. Intensive Care Med. 2003;31:1250-6.

22. Magiorakos AP, Srinivasan A, Carey RB, et al. Multidrug-resistant, extensively drug-resistant and pandrug-resistant bacteria: an international expert proposal for interim standard definitions for acquired resistance. Clin Microbiol Infect Mar. 2012;18(3):268-81.

23. Suwantarat N, Dumford DM, Ponce-Terashima $\mathrm{R}$, et al. Modification of antimicrobial prophylaxis based on rectal culture results to prevent fluoroquinolone-resistant Escherichia coli infections after prostate biopsy. Infect Control Hosp Epidemiol. 2013:34:973-6.

24. Summers SJ, Patel DP, Hamilton BD, et al. An antimicrobial prophylaxis protocol using rectal swab cultures for transrectal prostate biopsy. World J Urol. 2015;33:2001-7

25. Van Besien J, Uvin P, Van den Abeele AM, et al. Prevalence, risk factors, and clinical relevance of fluoroquinolone-resistant organisms in rectal cultures: should we target antibiotic prophylaxis prior to prostate biopsy? Adv Urol. 2016; doi:10.1155/2016/5392107.

26. Williamson DA, Freeman JT, Porter $\mathrm{S}$, et al. Clinical and molecular correlates of virulence in Escherichia coli causing bloodstream infection following transrectal ultrasound-guided (TRUS) prostate biopsy. J Antimicrob Chemother. 2013:68:2898-906.

27. Siriboon S, Tiengrim S, Taweemongkongsup $T$, et al. Prevalence of antibiotic resistance in fecal flora of patients undergoing transrectal ultrasoundguided prostate biopsy in Thailand. Urol Int. 2012;88:187-93.

28. Taylor S, Margolick J, Abughosh Z, et al. Ciprofloxacin resistance in the faecal carriage of patients undergoing transrectal ultrasound guided prostate biopsy. BJU Int. 2013;111:946-53.

29. Minamida S, Satoh T, Tabata $K$, et al. Prevalence of fluoroquinolone-resistant Escherichia coli before and incidence of acute bacterial prostatitis after prostate biopsy. Urology. 2011;78:1235-9.

30. Chrisofos M, Papatsoris AG, Dellis A, Varkarakis IM et al. Can prostate biopsies affect erectile function? Andrologia. 2006;38:79-83. 
31. Batura D, Rao GG, Nielsen PB. Prevalence of antimicrobial resistance in intestinal flora of patients undergoing prostatic biopsy: implications for prophylaxis and treatment of infections after biopsy. BJU Int. 2010;106:1017-20

32. Steensels D, Slabbaert K, De Wever L, et al. Fluoroquinolone-Resistant E. Coli in intestinal flora of patients undergoing transrectal ultrasound-guided prostate biopsy-should we reassess our practices for antibiotic prophylaxis? Clin Microbiol Infect. 2012;18:575-81.

Submit your next manuscript to BioMed Central and we will help you at every step:

- We accept pre-submission inquiries

- Our selector tool helps you to find the most relevant journal

- We provide round the clock customer support

- Convenient online submission

- Thorough peer review

- Inclusion in PubMed and all major indexing services

- Maximum visibility for your research

Submit your manuscript at www.biomedcentral.com/submit
Biomed Central 